\title{
Dynamic and Geometrical Models to Estimate the Density of Fully-Loaded Bubbles in the Column Flotation of Silica Sand
}

\author{
Alejandro Uribe-Salas*, Roberto Pérez-Garibay and Fabiola Nava-Alonso
}

CINVESTAV-IPN Unidad Saltillo, Ramos Arizpe, 25900 Coah., México

\begin{abstract}
This paper presents a comparison between the density of the bubble-particle aggregate pertinent to column flotation of mineral particles estimated with two models: one resulting from a balance of the forces acting on the aggregate that rises in a quiescent aqueous solution (termed dynamic or hydrodynamic model), and the other, of geometrical nature, which consists in accommodating the particles of known density, morphology and size distribution on the surface of an spherical bubble of known diameter. To compare the performance of the models, knowledge of terminal velocity $\left(V_{t}\right)$, diameter $\left(d_{b p}\right)$ and friction coefficient $\left(C_{d}\right)$ of the aggregate is required, as well as the size distribution of particles adhered to the bubbles, in order to determine the density of the aggregate $\left(\rho_{b p}\right)$. It is concluded that the geometrical model is able of approximating the estimations of the dynamic model, and may be therefore used to preliminary estimate the maximum density that a bubble-particle aggregate may reach.
\end{abstract}

\section{INTRODUCTION}

Froth flotation is currently the main available technology to industrially separate particles of minerals (and other materials), and to produce high grade concentrates. Due to its versatility, besides the traditional application in the concentration of sulfides and industrial minerals (coal, barite, fluorite, etc.), at present its use has been extensively adopted by a wide variety of industries such as printed paper recycling, waste water treatment, to name a few. Currently, froth flotation has become an advanced technology which is used to process two billion tonnes of solids annually [1].

Nevertheless, the phenomena that occur in the collection zone of mechanical cells and columns have not yet been completely understood, due to the lack of sensors capable of measuring parameters such as the velocity and degree of mineralization of the bubble-particle aggregate, gas holdup distribution, etc. Of particular interest is the density of the bubble-particle aggregate (e.g., the air bubble partially (or fully) covered by particles), which may reach values close to that of the collection zone, thus lacking of buoyancy to rise to the froth phase. Fundamental work reported in the literature [2] on bubble loading under controlled conditions, have shown relatively large bubbles (3-6 mm diameter) might be completely covered by particles. As well, other authors [3] have reported that in the froth zone of industrial cells, bubbles are almost fully loaded by particles. To this regard, the present authors [4] have shown that under certain conditions, the column flotation of a zinc concentrate (sphalerite, $d_{80}=105 \mu \mathrm{m}$ ), may give rise to fully-loaded bubbles which reach density values close to that of water, therefore

*Address correspondence to this author at the Add/ Affiliation CINVESTAV-IPN Unidad Saltillo, Ramos Arizpe, 25900 Coah. Mexico; Tel 52(844) 438-9600 ext. 9644; Fax 52(844) 438-9610;

E-mail: alejandro.uribe@cinvestav.edu.mx, alejandro.uribe@prodigy.net.mx risking the possibility of being dragged by the down-flowing slurry to the tailings discharge. Similar phenomena were also observed by the authors in the flotation of silica sand [5].

According to the above, the aim of the work presented in this paper is to develop and validate a model to accommodate the particles of a known size distribution and morphology on the entire surface of a bubble of known diameter so the density of the resulting bubble-particle aggregate may be estimated. To evaluate the performance of the model, termed geometrical model, their estimations are compared with the computations of a model based on the balance of the forces acting on the aggregate, which is solved with the aid of experimental measurements of terminal velocity and diameter of the aggregate.

\section{HYDRODYNAMIC MODEL FOR BUBBLE LOADING}

In order to determine the bubble diameter that determines if the fully-loaded bubble rises or sinks in a quiescent aqueous medium, a modified laboratory-scale flotation column was designed and built (Fig. 1) which allows to measure both the diameter and velocity of the bubble completely covered by silica sand of known particle size distribution, when it rises in a quiescent aqueous solution. The measurements are then used to estimate the density of the bubbleparticle aggregate, based on the equation of the terminal velocity that results from a balance of forces acting on the aggregate [6]):

$$
V_{t}=\left[\frac{4}{3} \frac{g d_{b p}}{C_{d}}\left(\frac{\rho-\rho_{b p}}{\rho}\right)\right]^{1 / 2}
$$

where $V_{t}, d_{b p}$ and $\rho_{b p}$ are the terminal velocity (m/s), diameter $(\mathrm{m})$ and density $\left(\mathrm{kg} / \mathrm{m}^{3}\right)$ of the bubble-particle aggregate, respectively; $\rho$ is the density $\left(\mathrm{kg} / \mathrm{m}^{3}\right)$ of the fluid; $g$ is the acceleration due to gravity $\left(9.81 \mathrm{~m} / \mathrm{s}^{2}\right)$, and $C_{d}$, the friction 
coefficient, is given by one of the available correlations, for example that presented in the literature [7]:

$$
C_{d}=0.28 \times\left(1+\frac{9.06}{R e_{b p}^{1 / 2}}\right)^{2}
$$

with the Reynolds number of the aggregate $\left(R e_{b p}\right)$ given by:

$$
R e_{b p}=\frac{d_{b p} V_{t} \rho}{\mu}
$$

where $\mu$ is the viscosity ( $\mathrm{kg} / \mathrm{m} \mathrm{s}$ ) of the fluid.

It is important to note that in Equation (1) the terminal velocity is positive when the bubble-particle aggregate rises and that the drag force acts opposite to the movement direction of the aggregate.

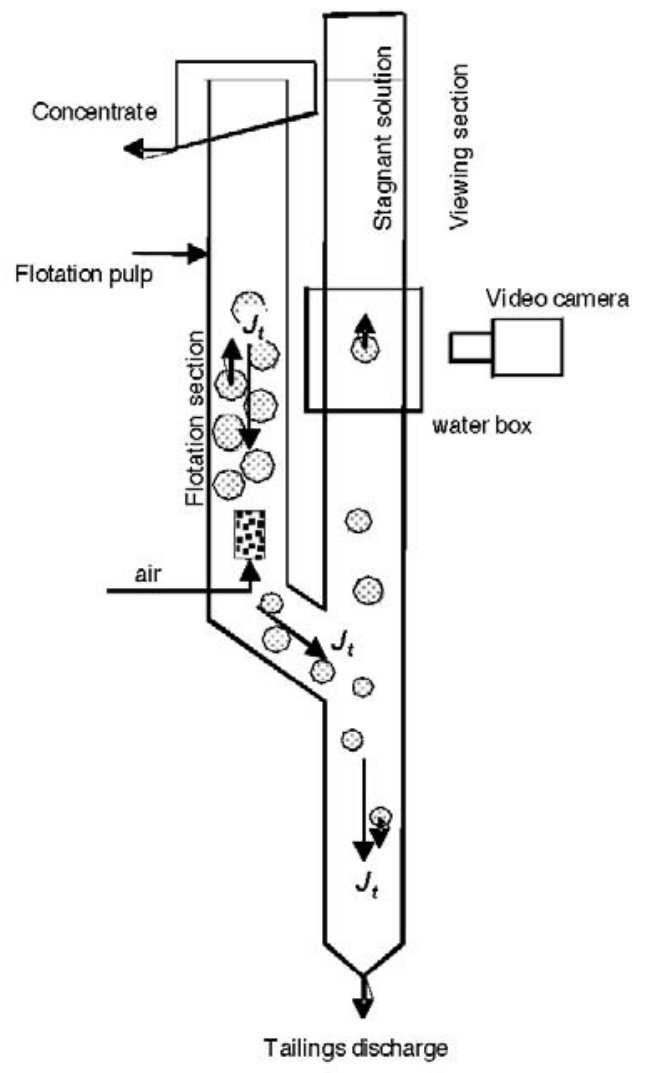

Fig. (1). Schematic of the modified flotation column used in this work, which illustrates the phenomenon under study and the experimental methodology used to measure the diameter and velocity of the bubble-particle aggregates that are dragged to the tailings discharge. ( $J_{t}$ is the slurry superficial rate, that is, the volumetric slurry flow rate divided by the cross-sectional area of the column).

\section{GEOMETRICAL MODEL FOR BUBBLE LOADING}

The model is based on the loading of a gas bubble with a size distribution of spherical particles. The model considers a mass of particles $M_{t}$ of density $\rho_{p}$, completely covering the surface area of the bubble (e.g., $\phi=1$ ), and that due to the presence of a size distribution of particles which efficiently fills the space (volume or area), the fraction of bubble surface area actually occupied by particles $(\varepsilon)$ is close to unity.
To this respect, the present authors have performed experimental measurements of $\varepsilon$ using rounded silica particles of relatively coarse size distribution, and a value ca. 0.8 have been estimated [8]. In the present study values of $\varepsilon$ equal $0.78,0.92$ and 1 are tested, according to considerations of Fig. 2). As well, the model assumes that a particle attaches to the bubble surface with a contact angle of $50^{\circ}$, which implies that the particle appears to enter the bubble one fifth of its diameter, as illustrated in Fig. (3). A contact angle of $50^{\circ}$ was selected since, as reported in the literature (Dippenaar IJMP 1982) [9], highly hydrophobic particles, characterized by contact angles of $90^{\circ}$ and higher, enhance bubble coalescence.
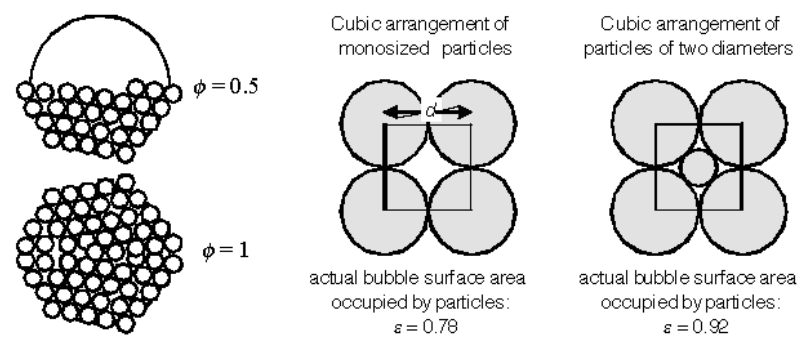

Fig. (2). Fraction of bubble surface area that adheres particles $(\phi)$ and fraction of bubble surface area actually occupied by particles $(\varepsilon)$, when spherical particles are accommodated in a square array on the bubble surface (note that for a size distribution of particles $\varepsilon$ $\rightarrow 1)$.

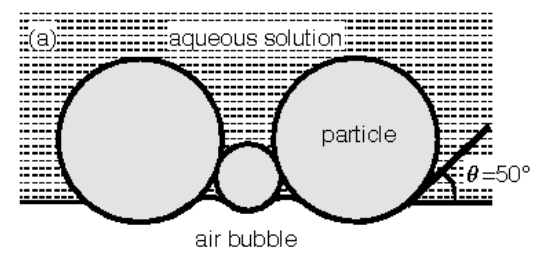

(b)

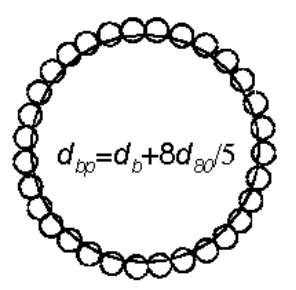

Fig. (3). Contact angle of $50^{\circ}$ between particles and bubbles considered in the geometrical model, typical of moderately hydrophobic particles. It is proposed that the diameter of the particles seating on the bubble surface is the $d_{80}$ of the distribution.

The use of the model consists in accommodating a mass $M_{t}$ of particles of the known size distribution on the surface of a bubble of diameter $d_{b}$ which is completely covered by particles (e.g., $\phi=1$ ) with a packing factor close to unity $(\varepsilon=0.78,0.92$ and 1$)$, to produce a bubble-particle aggregate having a density given by:

$$
\rho_{b p}=\frac{M_{t}}{\frac{\pi}{6} d_{b}^{3}+\frac{M_{t}}{\rho_{p}}}
$$


The steps follow in the development of the model are:

1.- The size distribution of particles in Fig. (4) are expressed in terms of size fraction discrete approximations in order to define de arithmetic mean $d_{i}$ as the average diameter of the size fraction $+d_{n} /-d_{n+1}$, and $m_{i}$ as the mass fraction of the particles in the size class.

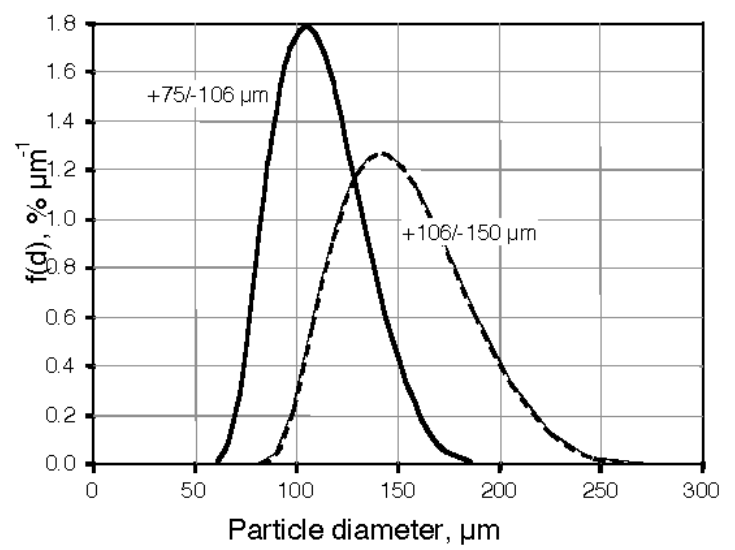

Fig. (4). Mass density function [10] of the silica sand used in this work, obtained with a Coulter LS100Q particle size analyzer based on laser diffraction techniques. The 80 percent passing size of the distributions $\left(d_{80}\right)$ are 118 y $161 \mu \mathrm{m}$, for the size fractions $+75 /-106$ and $+106 /-150 \mu \mathrm{m}$, respectively.

2.- The number of particles of average diameter $d_{i}$ present in the distribution is the ratio between the mass of particles with diameter $d_{i}\left(=m_{d i} \times M_{t}\right)$, and the mass of a particle of diameter $d_{i} \quad\left(=\frac{\pi}{6} d_{i}^{3} \times \rho_{p}\right)$, namely: $n_{d i}=\frac{m_{d i} \times M_{t}}{\frac{\pi}{6} d_{i}^{3} \times \rho_{p}}$

3.- The area occupied by the particles of diameter $d_{i}$ is the product of the number of particles and the cross-sectional area of a particle: $A_{d i}=n_{d i} \times \frac{\pi}{4} d_{i}^{2}$

4.- The area occupied by all the particles of the distribution is the summation of the areas occupied by the particles of the different diameters: $A_{p}=\sum_{i=1}^{n} A_{d i}$

5.- The diameter of the bubble $\left(d_{b}\right)$ whose surface area is fully covered by particles is estimated with the equation of the surface area of a sphere $\left(S_{b}=\pi d_{b}^{2}\right)$ :

$$
S_{b}=\pi d_{b}^{2}=\frac{A_{p}}{\varepsilon} ; \text { and } d_{b}=\sqrt{\frac{A_{p}}{\pi \varepsilon}}
$$

with $A_{p}=\frac{3}{2} \frac{M_{t}}{\rho_{p}} \times \sum_{i=1}^{n} \frac{m_{d i}}{d_{i}}$
6.- The volume of the particles $\left(V_{p}\right)$ is the ratio between their mass $\left(M_{t}\right)$ and density $\left(\rho_{p}\right)$, while the volume of the bubble $\left(V_{b}\right)$, is the volume of a sphere of diameter $d_{b}$.

7.- The diameter of the bubble-particle aggregate $\left(d_{b p}\right)$ is the bubble diameter plus $8 / 5$ the $\mathrm{d}_{80}$ of the particle size distribution (see Fig. 3):

$$
d_{b p}=d_{b}+\frac{8}{5} d_{80}
$$

It is considered that the $d_{80}$ of the size distribution is the most adequate diameter to represent the "visible diameter" of the distribution.

8.- Knowledge of $d_{b p}$ (and $d_{80}$ from the particle size distribution) allows estimation of $d_{b}$ with Equation (6), $A_{p}$ and $M_{t}$ with Equation (5), and finally $\rho_{b p}$ with Equation (4). The results obtained with the model are compared with the estimations of the hydrodynamic model in a $\rho_{b p}$ vs. $d_{b p}$ plot.

\section{EXPERIMENTAL PART}

\section{Apparatus}

Fig. (1) shows and schematic illustration of the modified flotation column used in this work, which consists of a 0.05 $\mathrm{m}$ diameter and $1.4 \mathrm{~m}$ height Plexiglas tube having a stainless steel porous sparger ( $5 \mu \mathrm{m}$ porous diameter) located in the bottom; this section is termed the "flotation section". Immediately below the sparger the column goes through a section of $0.15 \mathrm{~m}$ length inclined $135^{\circ}$ with respect to its longitudinal axis, which discharges into a second vertical section of $1.8 \mathrm{~m}$ height, which is opened in the top end and has the tailings port in the bottom. The upper part of the latter section is equipped with a water box which serves as a viewing window to video-recording the loaded bubbles rising in the still water that fills this section during column operation. The water box has been used in column flotation studies [11] to minimize the distortion of the bubbles caused by light refraction.

\section{METHODOLOGY}

The experimental methodology used in this work is described next.

1.- The conditioned slurry ( $p H$ 9.5, $340 \mathrm{~g} / \mathrm{t}$ dodecylamine, 15 ppm MIBC and $25^{\circ} \mathrm{C}$ ) is fed to the column close to the top of the flotation section and it is contacted with the swarm of air bubbles generated in the sparger $(\mathrm{Jg}=1.0$ $\mathrm{cm} / \mathrm{s}$ ). The slurry contains around $35 \% \mathrm{w} / \mathrm{w}$ of silica sand of known size distribution (Fig. 4). More details on the conditioning stage and column operation are reported elsewhere [5, 12].

2.- Hydrophobicity of silica particles is such that nearly full recovery is obtained.

3.- Mass flow rate of solids fed to the column is relatively high so most bubbles are completely loaded with particles.

4.- The relatively large bubbles of the size distribution have enough buoyancy to transport their load along the flotation section, to the concentrate launder. 
5.- The relatively small bubbles of the distribution lack of buoyancy to rise and are therefore dragged by the slurry to the tailings discharge.

6.- Bubbles of intermediate size are dragged by the descending slurry and are carried to the viewing section were they are able to rise in the stagnant solution that prevails above the discharge point; these bubbles are videorecorded when they pass through the water box. The diameter and velocity of the bubbles are obtained off-line from the video.

Fig. (4) presents the mass density function of the silica sand used in this work and Fig. (5) shows the rounded morphology of the particles which may be characterized by a sphericity shape factor of 0.82 [6].

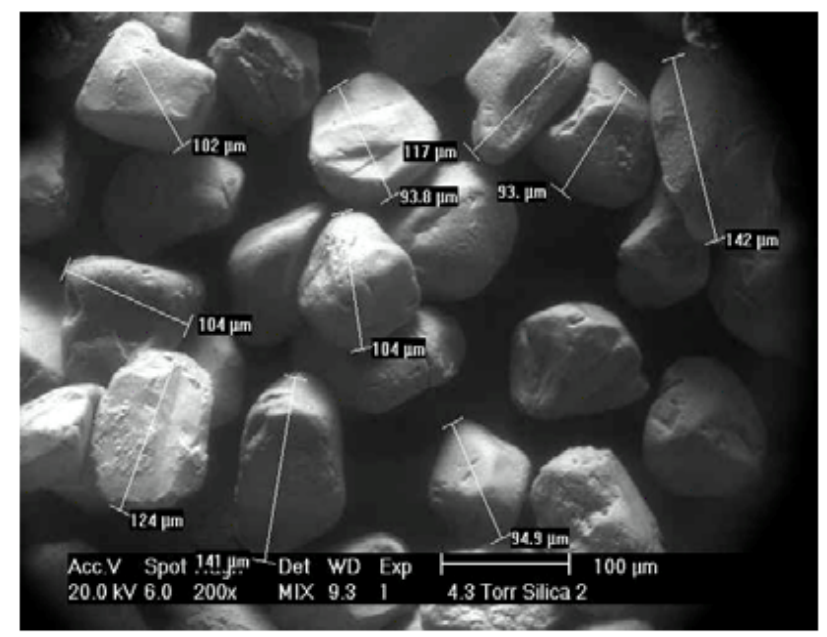

Fig. (5). Morphology of the silica sand used in this work, showing the rounded shape of the particles.

The mineralized bubbles were video recorded with a digital camera (Kodak ES-310) equipped with a Navitar 6000 macro lens. The images were processed with the aid of conventional image analysis software.

\section{RESULTS AND DISCUSSION}

Fig. (6) presents a typical photograph of a fully loaded bubble of around $1 \mathrm{~mm}$ diameter, slowly rising in the quiescent aqueous solution of the viewing section and Fig. (7) presents the experimental measurements of $V_{t}$ and $d_{b p}$ performed. It is apparent in the figure that for both particle size distributions tested, the rising velocity of the aggregate increases as their diameter increases; this is due to the fact that the buoyancy force overcomes the concomitant increase of the gravitational force. To better understand the results presented in the figure, let us consider a bubble of diameter $d_{b}$ which is completely covered with particles of the finer size distribution, and has a density close to unity, facing therefore difficulties to rise in the viewing section; consequently, a bubble of the same diameter but loaded with particles of the coarser distribution will have a density above unity, sinking in the viewing section and leaving the column in the tailings discharge. This is the reason of having video-recorded aggregates covered with the finer particles of smaller diameters compared to those covered with the coarser particles; it is evident that both aggregates have similar rising velocities. It is worth to keep in mind that the aggregates rise in practically stagnant water, and are therefore free of convective currents produced by the slurry that flows downward in the flotation section, since the idea was to isolate and measure both their rising velocity and diameter.

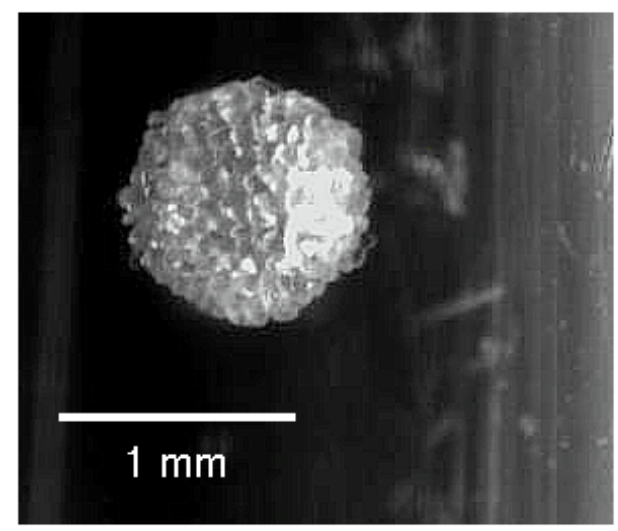

Fig. (6). Photograph of a bubble-particle aggregate completely loaded with silica particles of the coarser size distribution, rising in the quiescent aqueous solution of the viewing section.

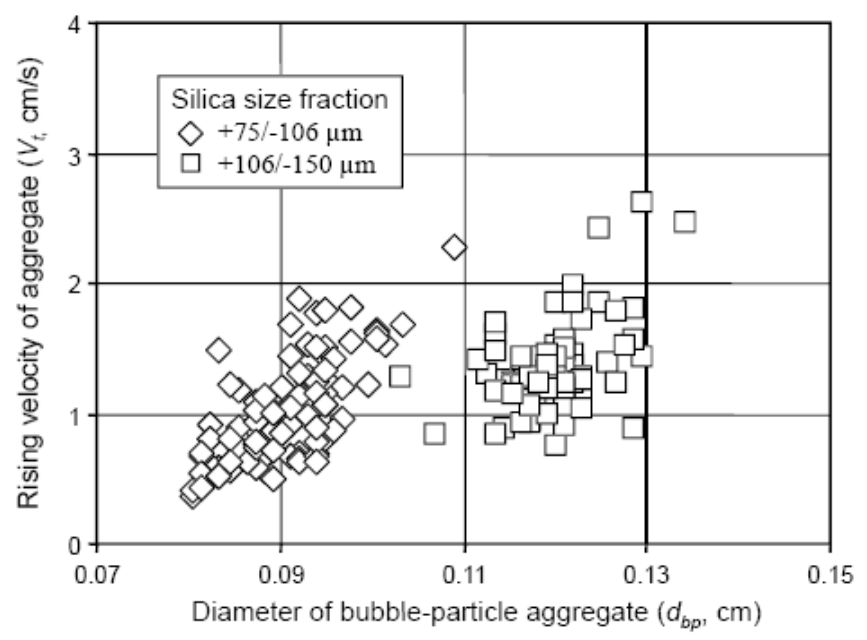

Fig. (7). (a) Terminal velocity of the bubble-particle aggregate $\left(V_{t}\right)$ vs. aggregate diameter $\left(d_{b p}\right)$, experimentally measured for the two silica size fractions tested.

Fig. (8) presents the relationship between $\rho_{b p}$ and $d_{b p}$ obtained with both the hydrodynamic model (Equations (1) to (3)), and the geometrical model (Equations (4) to (6)). In the figure the symbols are the estimates of the hydrodynamic model and the lines represent the estimates of the geometrical model: the dashed lines correspond to the density of the aggregate for the coarser distribution of particles $\left(d_{80}=161\right.$ $\mu \mathrm{m})$ and the full lines are the aggregate density for the finer fraction $\left(d_{80}=118 \mu \mathrm{m}\right)$. As mentioned above, the particle appears to penetrate the bubble approximately $1 / 5$ of its diameter. Packing fractions of 0.78 to 1 are compared.

It is clearly observed in Fig. (8) that the geometrical model describes well the predictions of the hydrodynamic model, particularly when packing factors $(\varepsilon)$ of 0.92 and 1 are considered. This suggests that the assumptions used in the model development are a good approximation to the ac- 
tual situation, particularly when rounded particles, close to spherical, are floated. According to the above, the model may be of use to predict the behavior of all the bubbles of a particular size distribution, that are contacted with a size distribution of floating particles under conditions of solids overcharging (e.g., relatively high mass flow rate of solids in the feed to the cell), which is usually the case of the final cleaning stages of heavy minerals such as base-metal sulfides. This will allow having a preliminary approximation of the minimum volumetric flow rate of air required to safely float all the hydrophobic particles present in the flotation pulp.

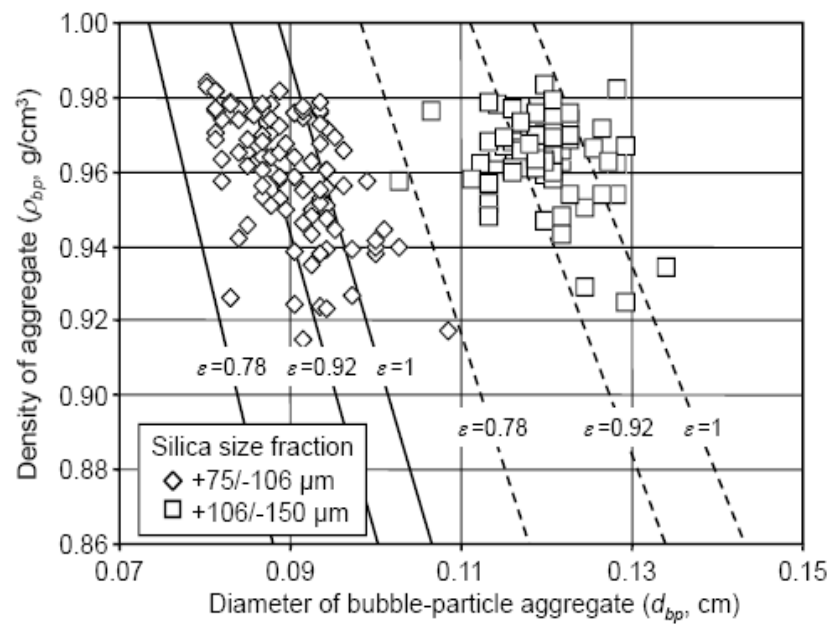

Fig. (8). Comparison between the relationship $\rho_{b p}$ vs. $d_{b p}$ obtained with the hydrodynamic model (symbols) and the geometrical model: full lines for the $+75 /-106 \mu \mathrm{m}$ size fraction and dashed lines for the $+106 /-150 \mu \mathrm{m}$ size fraction.

\section{CONCLUSIONS}

The experimental apparatus designed and built and the experimental methodology developed allowed to isolate and video-record fully-loaded air bubbles with silica sand of rounded morphology, with the aim of measuring both their diameter and rising velocities in a quiescent aqueous solution.

It was observed that the terminal velocity of the bubbleparticle aggregate increased as its diameter increased, and that for a given diameter, the velocity of an aggregate loaded with the coarser particle distribution $(+106 /-150 \mu \mathrm{m})$ was substantially smaller than that of an aggregate loaded with the finer distribution $(+75 /-106 \mu \mathrm{m})$.

The equation that resulted from the balance of the forces acting on the bubble-particle aggregate allowed to predict the density of the fully-loaded bubble, based on the experimental measurements of its diameter and rising velocity.

The geometrical model developed permitted to obtain $\rho_{b p}$ vs. $d_{b p}$ relationships that approximate those obtained with the dynamic model, thus suggesting that the assumptions made described well the actual bubble-particle adhesion phenomena, thus providing a method to preliminary estimate the density of a bubble of certain diameter fully loaded with a specific size distribution of hydrophobic particles.

\section{ACKNOWLEDGEMENTS}

Thanks are due to Ms. Socorro García-Guillermo (B.Sc.), Mr. Antonio González-Anaya (M.Sc.) and Miss Elva Leticia Aguirre (B.Sc.) for their help in the laboratory and computing processing of the data. Financial support of CONACYT (México) is gratefully acknowledged.

\section{REFERENCES}

[1] A.J. Lynch, J.S. Watt, J.A. Finch, and G.J. Harbort, "History of Flotation Technology”, in Centenary of Flotation Symposium, pp. 1-15, 2005.

[2] R.P. King, T.A. Hatton, and D.G. Hulbert, "Bubble loading during flotation”, Trans. Inst. Min. Metall., vol. 83, pp. C112-C115, 1974.

[3] F. Gourram-Badri, P. Conil, and G. Morizot, "Measurements of selectivity due to coalescence between two mineralized bubbles and characterization of MIBC action on froth flotation”, Int. J. Miner. Process., vol. 51, pp. 197-208, October 1997.

[4] A. Uribe-Salas, R. Pérez-Garibay, and F. Nava-Alonso, “Operating parameters that affect the carrying capacity of column flotation of a zinc sulfide mineral”, Miner. Eng., vol. 20, pp. 710-715, June 2007.

[5] A. Uribe-Salas, P. de Lira, R. Pérez, F. Nava, L. Magallanes, and C. Lara, "Overloading of gas bubbles in column flotation of coarse particles and effect upon recovery”, Int. J. Miner. Process., vol. 71, pp. 167-178, September 2003.

[6] E.G. Kelly, and D.J. Spottiswood, Introduction to Mineral Processing. John Wiley \& Sons, 1982.

[7] F. Concha, and E.R. Almendra, "Settling velocities of particulate systems, 1. Settling velocities of individual spherical particles”, Int. J. Miner. Process., vol. 5, pp.349-367, February 1979.

[8] P.M. Gallegos-Acevedo, R. Pérez-Garibay, and A. Uribe-Salas, 2006. "Maximum bubble loads: Experimental measurement vs. analytical estimation”, Miner. Eng., vol. 19, pp. 12-18, January 2006.

[9] A. Dippenaar, "The destabilization of froth by solids. 1. The mechanism of film rupture”, Int. J. Miner. Process., vol. 9, pp. 1-14, January 1982.

[10] J.A. Herbst, "Rate processes in multiparticle metallurgical system,” in Rate Processes of Extractive Metallurgy, H.Y. Shon, and M.E. Wadsworth, Eds. New York: Plenum Press, pp. 53-160, 1979.

[11] J.B. Yianatos, J.A. Finch, G.S. Dobby, and M. Xu, "Bubble Size estimation in a bubble swarm”, J. Coll. Interface Sci., vol. 126, pp. 37-44, November 1988.

[12] R. Pérez-Garibay, P.M. Gallegos, A. Uribe, and F. Nava, "Effect of collection zone height and operating variables on recovery of overload flotation columns”, Miner. Eng., vol. 15, pp. 325-331, May 2002. 